\title{
Field Testing of ROCOF Algorithms in Multiple Locations on Bornholm Island
}

\author{
Paul S. Wright", Peter N. Davis ${ }^{*}$, Kevin Johnstone ${ }^{+}$, Gert Rietveld ${ }^{\dagger}$ and Andrew J. Roscoe ${ }^{+}$ \\ "National Physical Laboratory, UK \\ paul.wright@npl.co.uk
}

"NPL, UK; †VSL, Netherlands; + University of Strathclyde, UK

\begin{abstract}
The importance of rate of change of frequency (RoCoF) measurements in electrical power grids is discussed along with its measurement challenges. A measurement campaign in a renewable-rich grid to compare ROCOF measurement algorithms with a variety of configurations at multiple locations is presented. An example of a captured ROCOF event is given.

Index Terms - Phasor measurement units (PMU), Frequency measurement, Rate-of-Change-of-Frequency (ROCOF).
\end{abstract}

\section{RoCoF AS A METRIC IN RENEWABLE RICH GRIDS}

Network frequency and the rate-of-change-of-frequency (ROCOF) are key indicators of network stability and of the balance between electricity supply and demand. This balance is becoming more critical with the increased use of highlyvariable renewable energy sources (RES) for electricity generation, and at the same time present ROCOF measurements are inadequate for monitoring this balance.

Traditional electricity generation uses rotating machines which provide significant inertia to the grid, able to absorb unpredictable changes in consumer demand. They also act as voltage sources, mitigating unbalance and harmonics, leading to an almost "perfect" power quality (PQ). By contrast, RES are connected to the network via power-electronic converters, conventionally acting as current sources due to their inner control loops. These generally provide negligible contribution to grid inertia, and usually provide a negative or neutral contribution to PQ.

At their present levels, intermittent RES, connected as current sources, can be integrated into the power network with few problems, however as the proportion of RES and other new devices continues to grow, the aggregated effect of all the converters creates a worsening of $\mathrm{PQ}$ and a reduction of frequency/voltage stability. Eventually, a tipping point is reached where the entire power system becomes unviable and unstable, setting a limit on the growth of RES. To surpass this limit, a thorough re-evaluation of "Loss of Mains" (LOM) protection, converter control techniques, and generation control strategies is required. Central to this are reliable, accurate ROCOF measurements.

\section{RoCoF MEASUREMENT PRoBlems}

Unfortunately, the measurement of ROCOF is a significant problem with the poor PQ waveforms that particularly prevail in low-inertia power networks. This is because a ROCOF measurement first requires frequency to be found using the $d / d t$ derivative from phase, before ROCOF is itself determined via a second $d / d t$ derivative from frequency. Any noise on the original phase estimate due to poor $\mathrm{PQ}$, transients, fault events, or instrumentation noise are vastly "amplified" by the double derivative, causing spurious results.

RES distributed generators are protected using LOM relays, which trip when the indicated value of ROCOF exceeds a set threshold. However, during imperfect $\mathrm{PQ}$, the spurious noise/ripple uncertainty on ROCOF can be larger than the trip thresholds, resulting in false tripping, for which LOM relays are notorious. These false trips are highly undesirable because they are expensive to the operator and they stress other parts of the grid when major energy sources are disconnected.

Some algorithms for measuring ROCOF are much less tolerant of poor PQ than others. In particular, LOM relays and Phasor Measurement Units (PMU) from different manufacturers exhibit very different performance. The instrumentation used to measure ROCOF is overseen by the IEEE/IEC joint standards committee 60255-118-1. Due to the on-going difficulties with ROCOF, the committee has relaxed ROCOF compliance requirements during certain tests pending further research to determine algorithms that can operate reliably in representative use case scenarios that exist on power networks [1].

\section{ROCOF ALGORITHM TESTING ON BORNHOLM ISLAND}

Many researchers and manufacturers have already developed algorithms for ROCOF. The present research does not develop wholly new alternative algorithms from the ground up, rather the intention is to test and optimise those algorithms already developed. Whilst much can be learned by simulation testing of algorithms (as would be carried-out by algorithm developers), this research will be conducted in a real power network representative of the high RES penetrations typical of future power systems. This will help unlock the normative standardisation process by selecting the best algorithms, optimising their parameters, and understanding which real world events cause them to malfunction.

Bornholm Island is a power network situated in the Baltic Sea with a single interconnection to the Nordic Power Grid, largely capable of independent operation due to its high penetration of RES and an independent diesel power station 
for periods of low wind generation. Six metrology grade digitisers were installed on the Island in 2016 to conduct PQ research; a full description of the power network and the digitisers is given in [2]. These digitisers have the capability of remote reprogramming which here allows various ROCOF algorithms to be uploaded and reconfigured.

The six locations are chosen for distributed geographical coverage of the 588 sq.km island, but also for electrical interest. One instrument is located on the island interconnector and measures at the $60 \mathrm{kV}$ levels. A second instrument is connected at $10 \mathrm{kV}$ on the output of a wind farm. The remaining four instruments are at the low voltage (LV) $440 \mathrm{~V}$ level and comprise of the two main cities, a rural location and the LV side of the aforementioned wind park respectively.

All instruments are equipped with GPS which allows accurate time stamping of their measurements. ROCOF algorithms run in real-time and can be configured for various update rates ranging from one reading every $20 \mathrm{~ms}(50 \mathrm{~Hz}$ cycle period) to one reading per $100 \mathrm{~ms}$ giving a trade-off of measurement time latency and noise averaging. The underlying sample rate is adjustable and has a maximum of $30 \mathrm{kS} / \mathrm{s}$. When a ROCOF algorithm on any given instrument measures an absolute value above a preset threshold, a rolling buffer of raw waveform data before and after the trigger event is saved to the local hard disk. This data includes the three voltage and current phases together with detailed GPS timing information. This data can then be downloaded and examined.

Data from a ROCOF trigger event is potentially of significant interest; it could be representative of a real ROCOF event which would be potentially more frequent on a lowinertia network such as Bornholm. Alternatively, it could be a false trigger caused by a PQ disturbance or phase jump such as a switching transient, a voltage dip/swell, or unbalanced fault. Whichever the event, the response of the algorithm with its given configuration will provide valuable information on the limitations of ROCOF such as how PQ events can be rejected, whilst providing acceptable measurement update rates for real time power system protection. The ability to replay captured data through alternative algorithms and configurations will be highly advantageous to recommending new normative approaches to ROCOF measurements.

Simultaneous ROCOF measurements in multiple locations will also yield valuable information about the effects of different voltage levels and locations to the sensitivity of the measurement to spurious PQ events.

\section{PRELIMINARY RESULTS}

Up to now, two ROCOF algorithms have been implemented and uploaded to the instruments. The first algorithm is as recommended in the IEC/IEEE C37.118.1 standard [1] and the second algorithm is using continuously retuned filters as developed by the University of Strathclyde [3]. Measurements were started in December 2017 and using a
ROCOF update rate of $50 \mathrm{~Hz}$ and trigger setting of $2 \mathrm{~Hz} / \mathrm{s}$, an example ROCOF event was captured on 09/01/18 as shown in Fig.1. The $\sim 40 \mathrm{~Hz} / \mathrm{s}$ event is associated with an unbalanced fault lasting some 4 cycles.

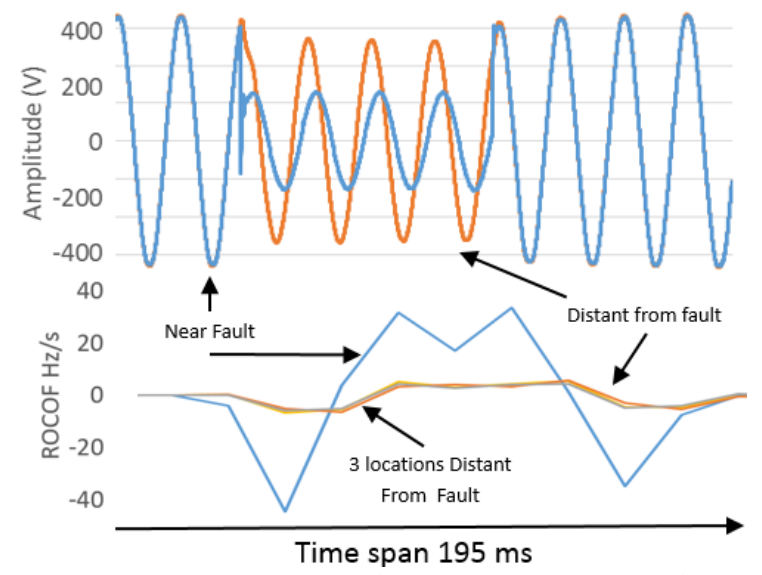

Fig.1, Top plot: phase L2 waveforms near and distant to a fault. Bottom plot: ROCOF as measured at the four locations.

The site presumed nearest to the fault clearly has the largest change in voltage and the largest ROCOF values.

Further results will be presented at the conference.

\section{CONCLUSION}

The measurement of ROCOF in a high-RES penetration electricity network will potentially reveal some of the typical scenarios that instruments and relays will be required to handle in future mainstream grids. It is anticipated that this work will provide essential data and knowledge to unlock the unsatisfactory normative standards situation for ROCOF and help set the boundary conditions for reliable measurements, whilst optimising the measurement algorithm parameters.

\section{ACKNOWLEDGEMENT}

The authors thank Andreas Christensen of Trescal DK and Lars and Poul Sonne-Pedersen from Energetic Bornholm for their technical support. The project work described in this paper has received funding from the EMPIR programme co-financed by the Participating States and the European Union's Horizon 2020 research and innovation programme.

\section{REFERENCES}

[1] "IEEE Std C37.118.1-2011 - IEEE Standard for Synchrophasor Measurements for Power Systems, IEEE, December 2011.

[2] P. S. Wright, A.E. Christensen, P. N. Davis and T. Lippert, "Multiple-Site Measurements of Harmonics for Analysis of Harmonic Propagation on Bornholm Island," IEEE Trans. Instrum. Meas., vol. 66, no. 6, pp. 1176 - 1183, March 2017.

[3] A. J. Roscoe, I. F. Abdulhadi and G. M. Burt, "P and M Class Phasor Measurement Unit Algorithms Using Adaptive Cascaded Filters," IEEE Trans. Power Delivery, vol. 28, no. 3, pp. 1447 1459, June 2013. 